\title{
Experimental bending fatigue data of additive-manufactured PLA biomaterial fabricated by different 3D printing parameters
}

\author{
Ali Dadashi ${ }^{1}$, Mohammad Azadi ${ }^{1 *}$ \\ 1 Faculty of Mechanical Engineering, Semnan University, Semnan, Iran; dadashi_ali@semnan.ac.ir and \\ m_azadi@semnan.ac.ir \\ * Correspondence: m_azadi@semnan.ac.ir; Tel.: +98-910-210-7280
}

\begin{abstract}
Abstract: Additive manufacturing (AM) are used in several industries such as automotive, aerospace, and medical sciences. One of the most common devices used in additive manufacturing is fused deposition modeling (FDM) 3D printers. This fabrication method has different inputs that affect the quality of the parts. In this research, the bending fatigue properties of polylactic acid (PLA) biomaterial made with a $3 \mathrm{D}$ printer are investigated. To demonstrate the influence of printing parameters on fatigue lifetime, standard specimens with nozzle diameters of 0.2-0.6 mm, extruder temperature of $180-240^{\circ} \mathrm{C}$, and print speed of $5-15 \mathrm{~mm} / \mathrm{s}$ were printed. After performing fully-reversed bending fatigue tests, it was found that printed specimens at $180^{\circ} \mathrm{C}$ have the best fatigue lifetime in most cases. Accordingly, fatigue behavior improved by reducing the nozzle diameter. Printing at lower temperatures also improved fatigue lifetime. The printing speed affected the slope of the S-N diagram, known as the fatigue strength exponent.
\end{abstract}

Keywords: Fatigue data; Polylactic acid; Additive manufacturing, 3D printing, Fused deposition modeling

\section{Introduction}

Polylactic acid (PLA) has been widely utilized in biomechanical applications besides other industries. Therefore, researchers have focused on the properties of the structure, fabricated by additive manufacturing (AM). Abeykoon et al. [1] investigated filling pattern, density, and speed effects on the mechanical, thermal, and morphological properties of the printed parts. For the influences of filling percentage, infill pattern, layer thickness, and extrusion temperature on mechanical properties of parts in fused deposition modeling (FDM), Alafaghani and Qattawi [2] used the design of experiments. Sandhu et al. evaluated the influence of slicing parameters on the surface roughness [3] and mechanical properties [4] of FDM prints. Travieso-Rodriguez et al. [5] demonstrated the fatigue behavior of PLA-wood composites produced by FDM. Patil et al. [6] provided a paradigm of multi-objective functions for optimizing FDM process parameters for printing PLA components.

Due to the effect of different 3D printing parameters on the parts fabricated by the FDM, it is necessary to design and optimize the polymer materials to fully comply with the mechanical requirements. Studies on the fatigue behavior of polymer materials are not sufficient and it is difficult to understand the role of fatigue in FDM-printed polymers [7]. Therefore, in this study, the effect of printing parameters, including print speed, nozzle diameter, and nozzle temperature, on the bending fatigue properties of standard PLA specimens were illustrated. The nozzle diameters were considered as $0.2-0.6 \mathrm{~mm}$, the extruder temperatures were selected as $180-240^{\circ} \mathrm{C}$, and the print speed was $5-15 \mathrm{~mm} / \mathrm{s}$. 


\section{Materials and Methods}

The standard dog-bone samples were fabricated according to ISO-1143 [8], as shown in Figure 1. In order to prepare fatigue test samples, an FDM printer was used (Figure 2). 3D printing was performed by transparent PLA filaments with a diameter of $1.75 \mathrm{~mm}$, made by YouSu Company.

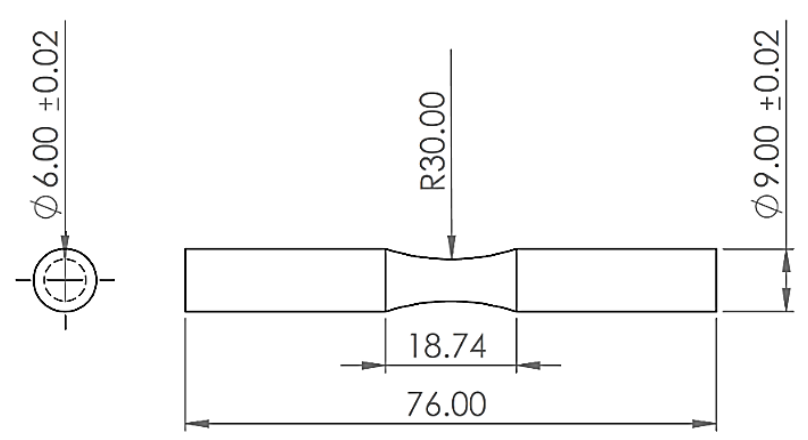

Figure 1. The geometry of the fatigue sample

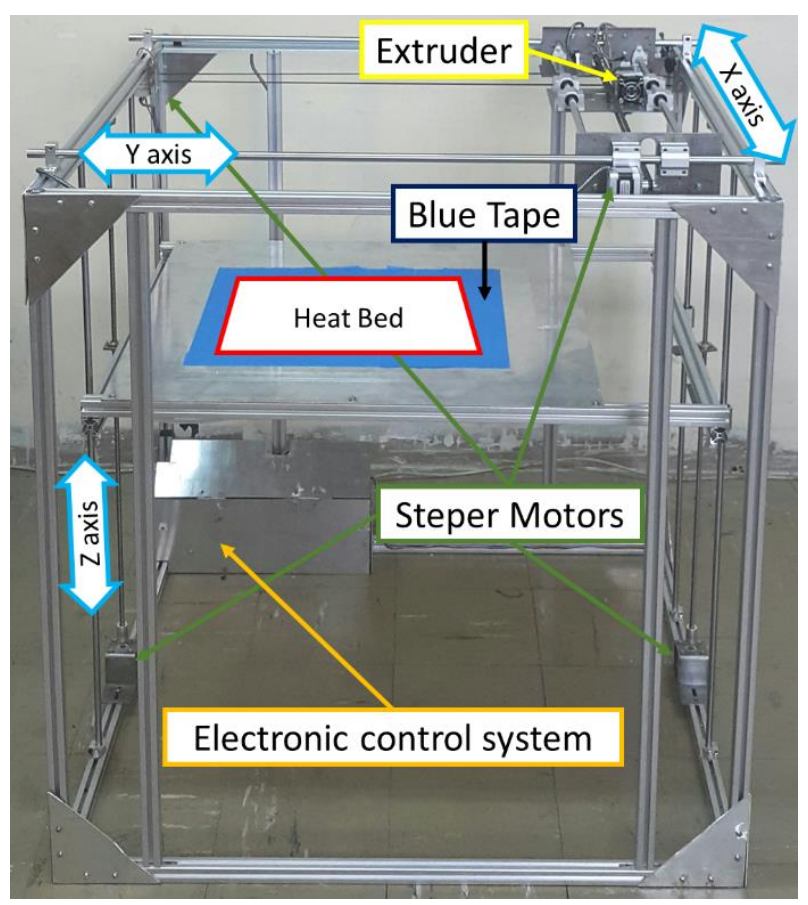

Figure 2. FDM 3D printer used for manufacturing the test specimens

Due to a large number of these parameters, the effect of three parameters, including print temperature, print speed, and nozzle diameter on fatigue lifetime, had been experimentally investigated at three different levels, based on Table 1, and other parameters were constant, according to Table 2 . Bending fatigue tests were performed under fullyreversed cyclic loads through a high-cycle fatigue regime with the rotating bending fatigue testing machine at room temperature with a frequency of $100 \mathrm{~Hz}$. This test device, made by Santam Company, is shown in Figure 3.

Table 1. Variable 3D printing parameters

\begin{tabular}{ccccc}
\hline Parameters & Dimension & Low Level & Medium Level & High Level \\
\hline Print Speed & $\mathrm{mm} / \mathrm{s}$ & 5 & 10 & 15 \\
Print Temperature & ${ }^{\circ} \mathrm{C}$ & 180 & 210 & 240 \\
Nozzle Diameter & $\mathrm{mm}$ & 0.2 & 0.4 & 0.6 \\
\hline
\end{tabular}


Table 2. Constant 3D printing parameters

\begin{tabular}{ccc}
\hline Parameters & Dimension & Value \\
\hline Layer Thickness & $\mathrm{mm}$ & 0.2 \\
Perimeter & - & Top-1, Bottom-1 \\
Solid Layers & - & Rectangular \\
Fill Pattern & - & 30 \\
Travel Speed & $\mathrm{mm} / \mathrm{s}$ & 30 (Room Temperature) \\
Bed Temperature & ${ }^{\circ} \mathrm{C}$ & Horizontal \\
Print Direction & - & 60 \\
Infill & $\%$ & r
\end{tabular}

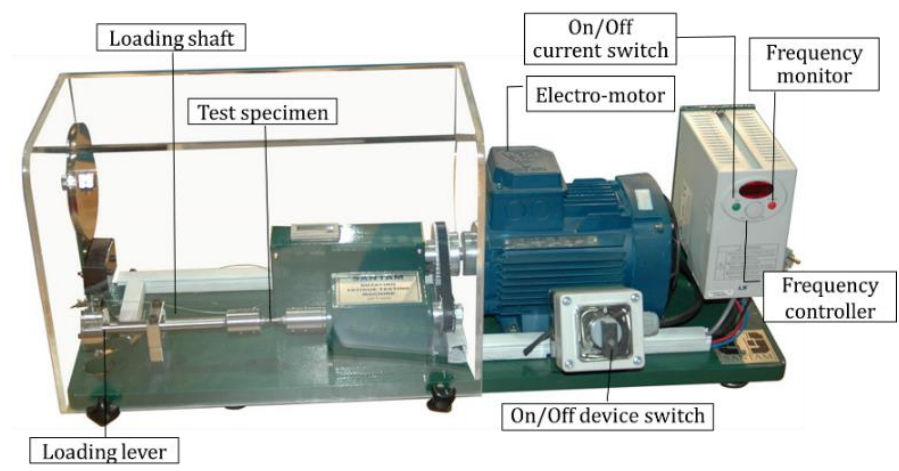

Figure 3. SFT-600 Santam rotating bending fatigue testing device

\section{Results and Discussion}

Fatigue test data are often presented in curves of the stress versus the number of cycles to failure, while the logarithmic scale is used for the lifetime. To compare the results, data of different 3D printing temperatures and speeds with a constant nozzle diameter were drawn in a single plot. Moreover, based on researches previously conducted in the research laboratory of Advanced Materials Behavior (AMB), the Faculty of Mechanical Engineering, Semnan University, three data sets on 3D-printed PLA are presented in all diagrams to compare the results (Figures 4-6). The print parameters in these results are summarized in Table 3.

Table 3. 3D printing parameters of researches in the Research Laboratory of AMB

\begin{tabular}{|c|c|c|c|c|}
\hline Parameters & Dimension & AMB Lab 1 & AMB Lab 2 & AMB Lab 3 \\
\hline Material (Filament Color) & - & PLA (Black) & PLA (Black) & PLA (White) \\
\hline Filament Diameter & $\mathrm{mm}$ & 1.75 & 1.75 & 1.75 \\
\hline Nozzle Diameter & $\mathrm{mm}$ & 0.4 & 0.4 & 0.5 \\
\hline Print Temperature & ${ }^{\circ} \mathrm{C}$ & 200 & 200 & 210 \\
\hline Print Speed & $\mathrm{mm} / \mathrm{s}$ & 50 & 50 & 50 \\
\hline Print Direction & - & Vertical & Horizontal & Horizontal \\
\hline Layer Thickness & $\mathrm{mm}$ & 0.15 & 0.15 & 0.2 \\
\hline Perimeter & - & 3 & 3 & 3 \\
\hline Solid Layers (Top, Bottom) & - & 3 & 3 & 3 \\
\hline Fill Pattern & - & Rectangular & Rectangular & Honeycomb \\
\hline Travel Speed & $\mathrm{mm} / \mathrm{s}$ & 60 & 60 & 80 \\
\hline Bed Temperature & ${ }^{\circ} \mathrm{C}$ & 60 & 60 & 50 \\
\hline Infill & $\%$ & 50 & 50 & 75 \\
\hline
\end{tabular}




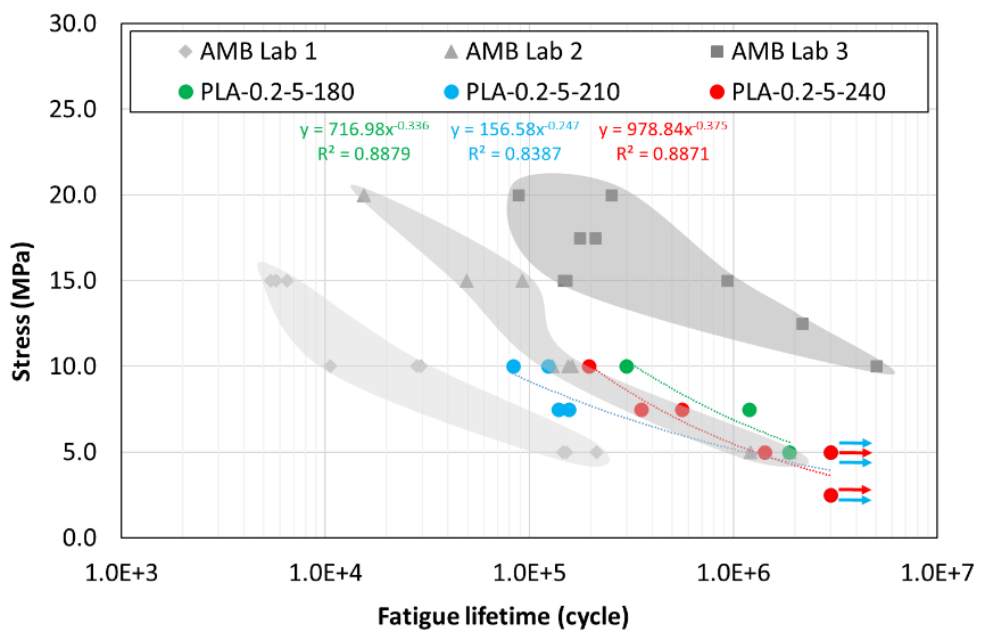

(a)

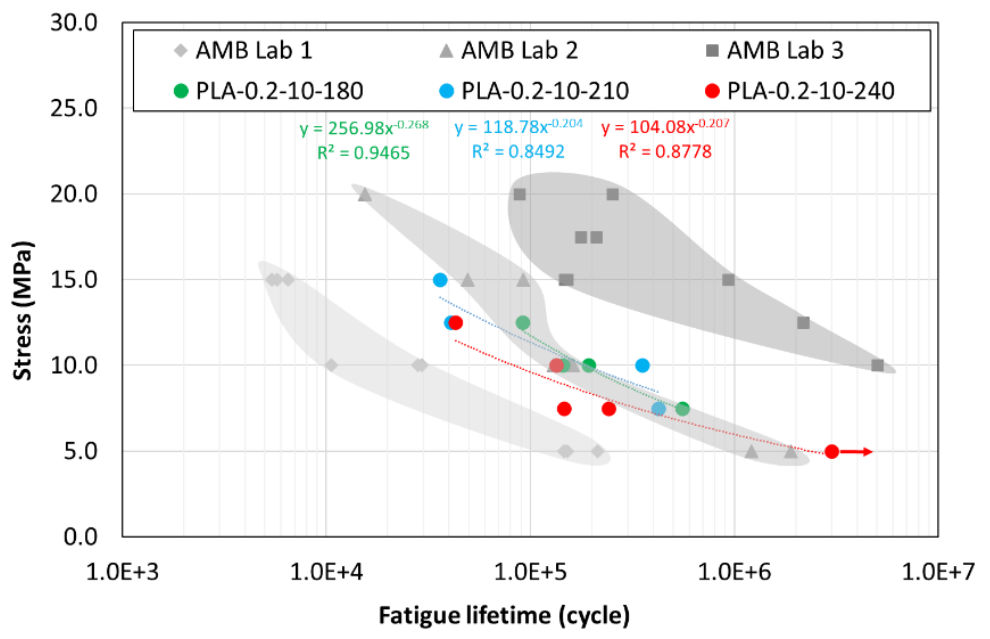

(b)

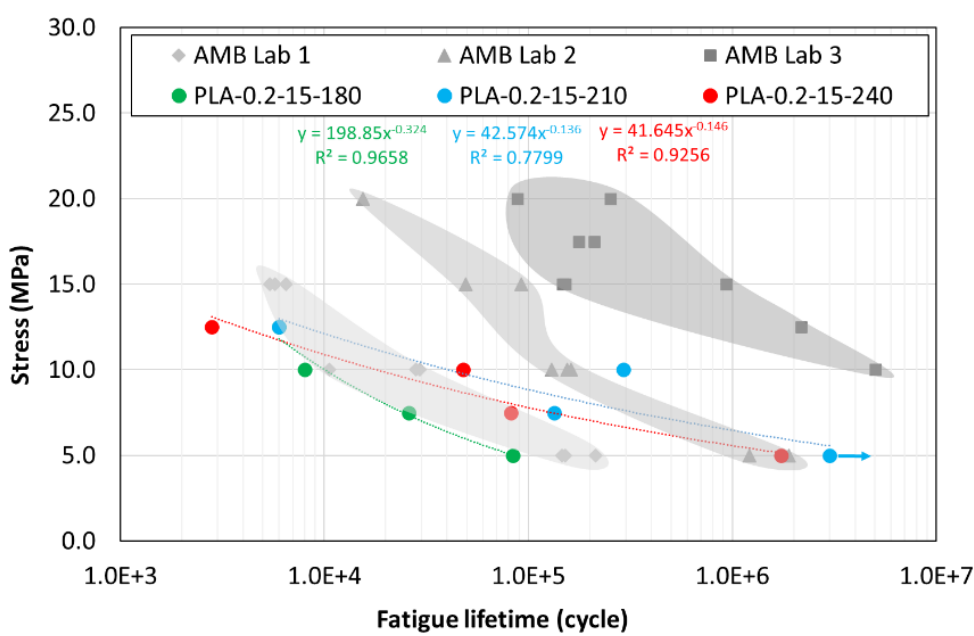

(c)

Figure 4. S-N diagrams for samples 3D-printed with $0.2 \mathrm{~mm}$ nozzle diameter at different speeds and temperatures 


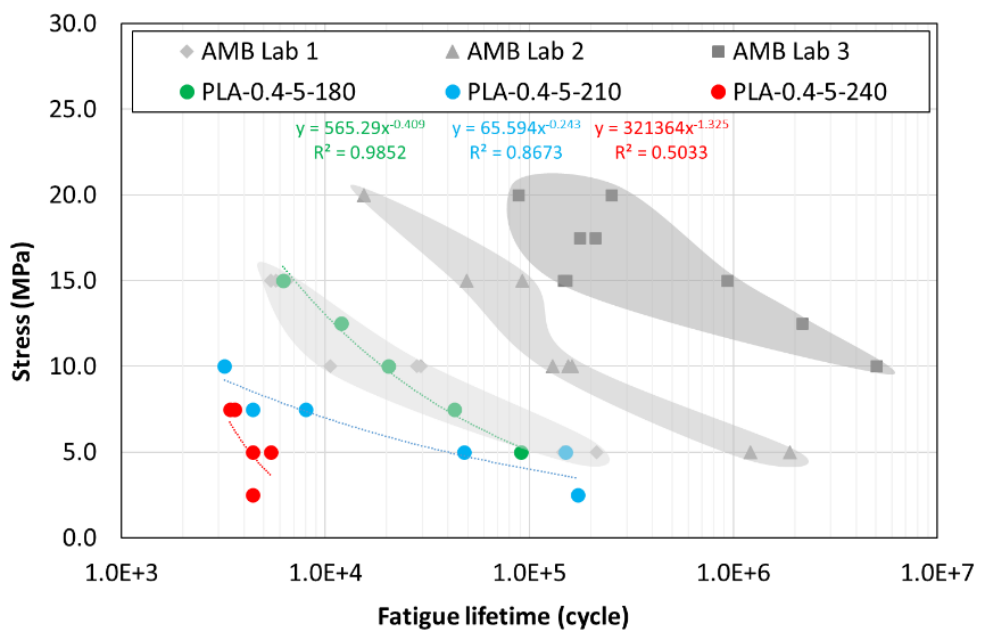

(a)

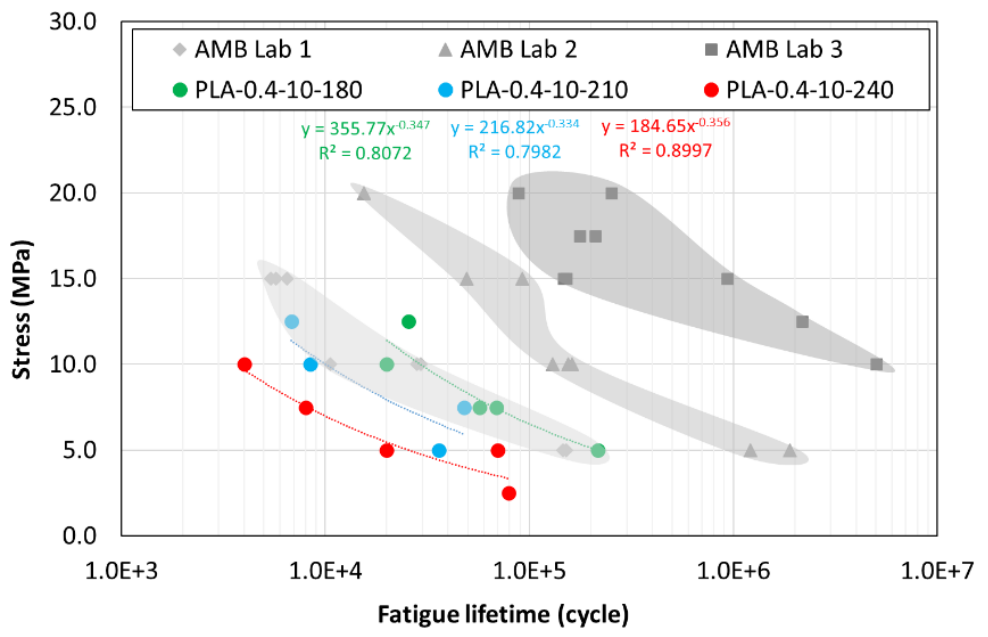

(b)

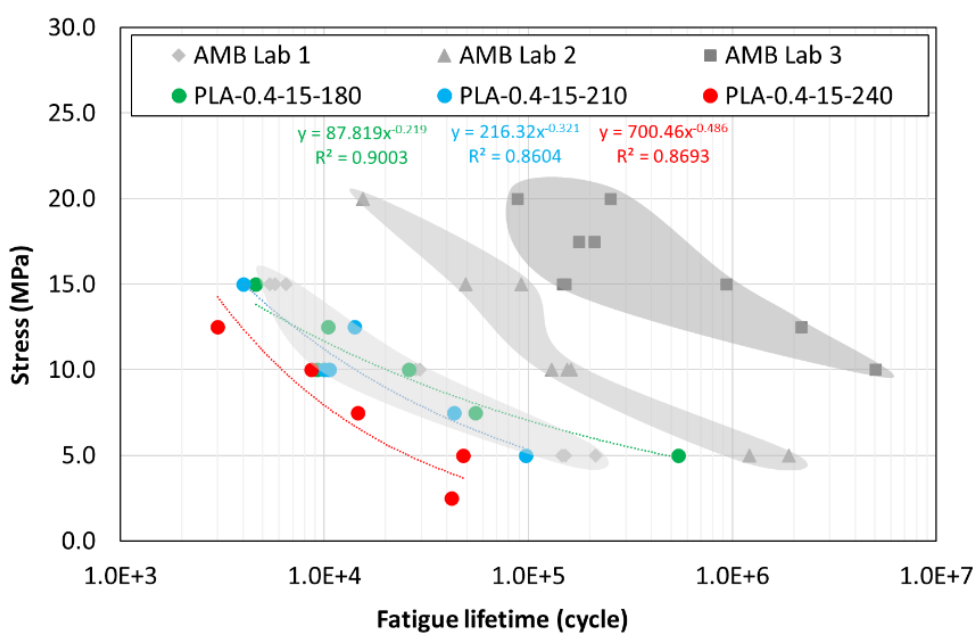

(c)

Figure 5. S-N diagrams for samples 3D-printed with $0.4 \mathrm{~mm}$ nozzle diameter at different speeds and temperatures 


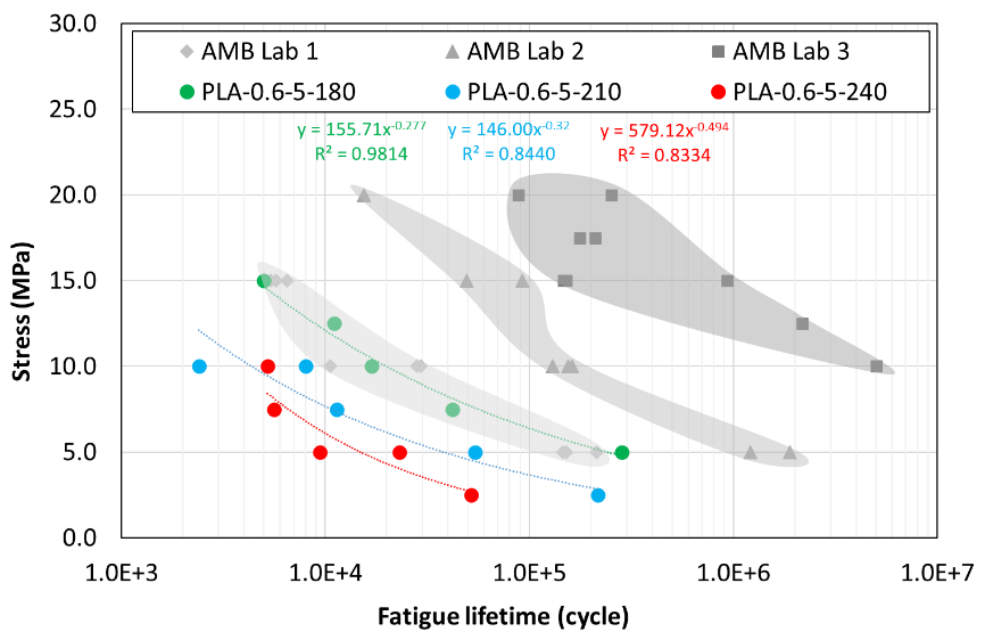

(a)

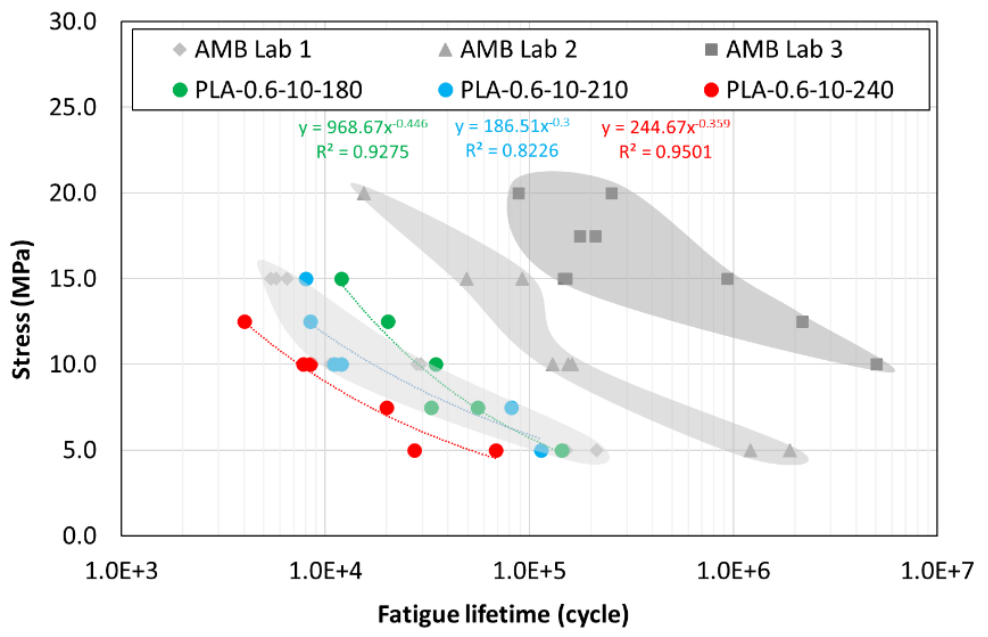

(b)

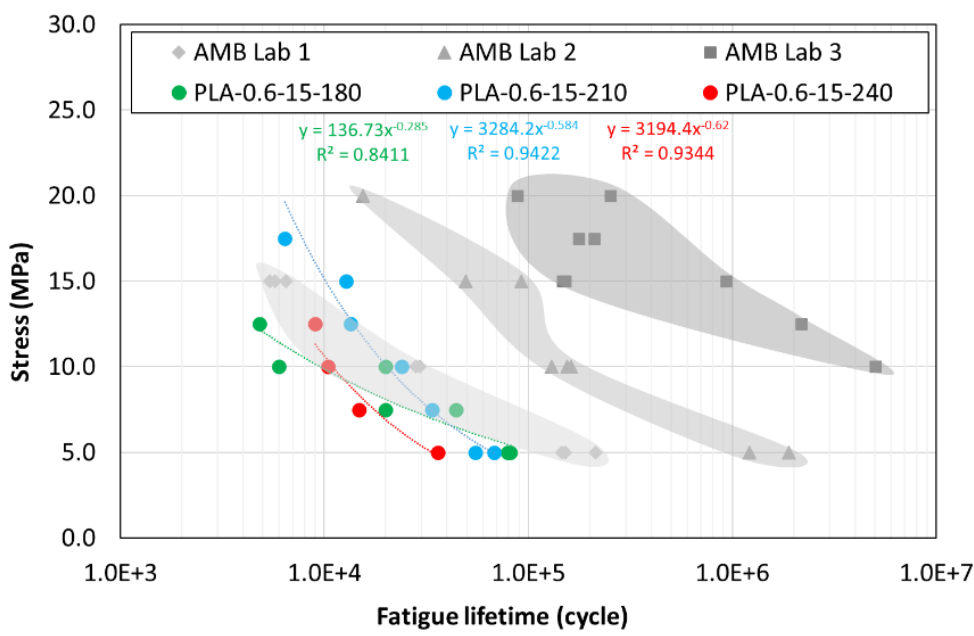

(c)

Figure 6. S-N diagrams for samples 3D-printed with $0.6 \mathrm{~mm}$ nozzle diameter at different speeds and temperatures

The results of the high-cycle fatigue tests showed that the samples printed at a temperature of $180{ }^{\circ} \mathrm{C}$ had a higher number of cycles to failure, in most cases. Therefore, the samples 3D-printed 
with a nozzle diameter of $0.2 \mathrm{~mm}$ with a printing speed of $5 \mathrm{~mm} / \mathrm{s}$ at a temperature of $180^{\circ} \mathrm{C}$ had the highest fatigue lifetime compared to other specimens. In contrast, the lifetime of printed samples with a nozzle diameter of $0.4 \mathrm{~mm}$ with a printing speed of $5 \mathrm{~mm} / \mathrm{s}$ and an extruder temperature of $240^{\circ} \mathrm{C}$ was about $34,000 \%$ shorter and these samples had the shortest lifetime. The smaller nozzle diameter improved the fatigue properties. The lifetime of the specimens decreased as the print temperature increased. In addition, increasing the speed of printing reduced the fatigue strength exponent. As a result, in samples 3D-printed with a $0.2 \mathrm{~mm}$ nozzle, the slope of the S-N plot at $240^{\circ} \mathrm{C}$ decreased by $61 \%$. Depending on the materials and conditions of use, the effect of the parameters varies and must be considered at the design stage. For example, higher printing speed improves tensile properties while temperature has been reported to have no effect on tensile properties [9].

\section{Conclusions}

In the present article, experimental bending fatigue data of additive-manufactured PLA biomaterial were presented. Then, highlighted results are obtained as follows:

- $\quad$ The decrease in printing temperature led to increasing the fatigue lifetime.

- The smaller the nozzle diameter, the longer the lifetime.

- Increasing the speed reduced the slope of the S-N diagram and thus affected the behavior through the low-cycle fatigue regime.

- $\quad 3 \mathrm{D}$ printing parameters could have different effects on the material behavior depending on the materials and conditions of use.

Supplementary Materials: The following supporting information can be downloaded at: https://data.mendeley.com/datasets/gyxsn7wg6c/1.

Author Contributions: Ali Dadashi: Formal analysis/ Investigation/ Data Curation/ Writing - Original Draft; Mohammad Azadi: Conceptualization/ Methodology/ Validation/ Investigation/ Resources/ Writing - Review \& Editing/ Visualization/ Supervision/ Project administration

Funding: The authors would like to acknowledge the financial support of the Ministry of Science, Research and Technology for this project under grant number 16-99-01-000194.

Institutional Review Board Statement: The study was conducted in accordance with the Declaration of Helsinki, and approved by the Institutional Review Board (or Ethics Committee) of Semnan University."

Informed Consent Statement: Not applicable for this research due to have no human and animal issues.

Data Availability Statement: The data that support the findings of this article are available at Azadi, Mohammad; Dadashi, Ali (2021), "HCF testing raw data on 3D-printed PLA polymers", Mendeley Data, V1, DOI: 10.17632/gyxsn7wg6c.1.

Acknowledgments: In this section, you can acknowledge any support given which is not covered by the author contribution or funding sections. This may include administrative and technical support, or donations in kind (e.g., materials used for experiments).

Conflicts of Interest: For this research, there is no conflict of interest for all authors. For any other conflict of interest, check the "Data Availability Statement" part.

\section{References}

1. Abeykoon, C.; Sri-Amphorn; P.; Fernando, A. Optimization of fused deposition modeling parameters for improved PLA and ABS 3D printed structures, International Journal of Lightweight Materials and Manufacture, 2020, 3, 3, 284-297.

2. Alafaghani, A.; Qattawi, A. Investigating the effect of fused deposition modeling processing parameters using Taguchi design of experiment method, Journal of Manufacturing Processes 2018, 36, 164-174.

3. Sandhu, G. S.; Boparai, K. S.; Sandhu, K. S. Effect of slicing parameters on surface roughness of fused deposition modeling prints, Materials Today: Proceedings 2021, 48, 1339-1345.

4. Sandhu, G. S.; Boparai, K. S.; Sandhu, K. S. Influence of slicing parameters on selected mechanical properties of fused deposition modeling prints, Materials Today: Proceedings 2021, 48, 1378-1382.

5. Antonio Travieso-Rodriguez, J.; Zandi, M. D.; Jerez-Mesa, R.; Lluma-Fuentes, J.; Fatigue behavior of PLA-wood composite manufactured by fused filament fabrication, Journal of Materials Research and Technology 2020, 9, 4, 8507-8516.

6. Patil, P.; Singh, D.; Raykar, S. J.; Bhamu, J. Multi-objective optimization of process parameters of Fused Deposition Modeling (FDM) for printing Polylactic Acid (PLA) polymer components, Materials Today: Proceedings 2021, 45, 4880-4885. 
7. Shanmugam, V.; Das, O.; Babu, K.; Marimuthu, U.; Veerasimman, A.; Johnson, D. J.; Neisiany, R. E.; Hedenqvist, M. S.; Ramakrishna, S.; Berto, F. Fatigue behavior of FDM-3D printed polymers, polymeric composites and architected cellular materials, International Journal of Fatigue 2021, 143, 106007.

8. ISO 1143, Metallic materials - Rotating bar bending fatigue testing, ISO International Standard 2010.

9. Ansari, A. A.; Kamil, M.; Effect of print speed and extrusion temperature on properties of 3D printed PLA using fused deposition modeling process, Materials Today: Proceedings 2021, 45, 5462-5468. 